\title{
Métodos de medição de altura em fragmento de Floresta Estacional Decidual
}

\author{
Lucas Matias de Souza FRUTUOSO¹, Débora de Melo ALMEIDA², João Gilberto Meza UCELLA FILHO3*, \\ Vital Caetano BARBOSA JUNIORํ, Gabriel Siqueira de ANDRADE¹, Juliana Lorensi do CANTO1
}

\author{
${ }^{1}$ Escola Agrícola de Jundiaí, Universidade Federal do Rio Grande do Norte, Macaíba, RN, Brasil. \\ (Orcid: 0000-0001-9935-9766; 0000-0001-6102-2631; 0000-0002-4612-7539; 0000-0003-1551-1543) \\ 2Pós-Graduação em Ciências Florestais, Universidade Federal Rural de Pernambuco, Recife, PE, Brasil. (Orcid: 0000-0002-3336-5044) \\ 3 Pós-Graduação em Ciência e Tecnologia da Madeira, Universidade Federal de Lavras, Lavras, MG, Brasil. \\ *E-mail: 16joaoucella@gmail.com (Orcid: 0000-0001-9797-3332)
}

Recebido em 10/05/2020; Aceito em 08/09/2020; Publicado em 25/09/2020.

\begin{abstract}
RESUMO: Dificuldades relativas à aquisição e à utilização de hipsômetros contribuem para o uso da estimativa visual da altura de árvores em florestas nativas. Diante disso, este trabalho objetivou comparar o método da estimativa visual com balizamento com o hipsômetro digital Haglof na medição de altura de árvores em um fragmento de Floresta Estacional Decidual. A coleta de dados compreendeu 187 árvores contidas em quatro parcelas permanentes. Para as análises comparativas, os dados foram distribuídos em classes de altura e de diâmetro. A estimativa visual com balizamento apresentou confiabilidade na medição da altura de árvores de até $11 \mathrm{~m}$, não diferindo estatisticamente do hipsômetro digital. Entretanto, observou-se uma tendência de subestimação da altura de árvores maiores. Para árvores com altura superior a $11 \mathrm{~m}$, o hipsômetro digital mostrou-se mais confiável.
\end{abstract}

Palavras-chave: inventário florestal; mensuração florestal; hipsômetro.

\section{Methods of measurement of height in fragment of decidual state forest}

\begin{abstract}
Difficulties related to the acquisition and use of hypsometers contribute to the use of the visual estimate of the height of trees in native forests. Therefore, this study aimed to compare the method of visual estimation with beaconing with the Haglof digital hypsometer to measure the height of trees in a fragment of Seasonal Deciduous Forest. The data collection comprised 187 trees contained in four permanent plots. For comparative analysis, the data were distributed in height and diameter classes. The visual estimation with beacon showed reliability in measuring the height of trees up to $11 \mathrm{~m}$, not differing statistically from the digital hypsometer. However, there was a tendency to underestimate the height of larger trees. For trees higher than $11 \mathrm{~m}$, the digital hypsometer was more reliable.
\end{abstract}

Keywords: forest inventory; forest measurement; hypsometer.

\section{INTRODUÇÃO}

A altura é uma importante característica da árvore e a sua determinação é fundamental para o cálculo de volume individual $e$, consequentemente, para a estimativa volumétrica de toda a floresta. Por isso, é uma variável importante no inventário e no manejo florestal (SILVA et al., 2017). Em florestas nativas, a altura das árvores ajuda também a compreender a estrutura vertical da comunidade, tendo importante significado ecológico, além de ser fundamental para fins de manejo florestal (SILVA et al., 2012a).

No entanto, a medição da altura total de uma árvore não é considerada uma tarefa fácil (SILVA et al., 2012a), podendo ser realizada de forma direta ou indireta. No método direto, a altura pode ser medida com trena a partir da derrubada da árvore; ou então, obtida por meio de uma régua ou vara altimétrica encostada na árvore em pé, dependendo da sua altura para ser viável; ou através de outros procedimentos mais difíceis de serem realizados, como por exemplo, a escalada da árvore (SILVA et al., 2012a). No método indireto, a altura pode ser obtida através da estimativa visual ou a partir de hipsômetros, instrumentos que se baseiam em princípios geométricos ou trigonométricos para a estimação da altura.
Entre os instrumentos mais utilizados, destacam-se os que se baseiam em princípios trigonométricos, que para serem utilizados corretamente, o operador precisa se localizar a uma distância conhecida da árvore, o que pode impedir a visualização da copa, principalmente, em florestas nativas, onde prevalece uma alta densidade de indivíduos e diversidade, principalmente, em florestas tropicais, tornando complexa a coleta dos dados de altura (GONÇALVES et al., 2009). Estimar com precisão a altura de árvores nesses ambientes, torna-se uma operação mais difícil, especialmente, para árvores mais altas, acarretando no aumento da chance de leituras equivocadas e intensificação dos erros (CURTO et al., 2013). Outros aspectos importantes quando se faz menção aos hipsômetros dizem respeito ao seu custo, à sua resistência ao uso diário e a sua facilidade ou não de manutenção (SILVA et al., 2019).

Em função disso, tornou-se cada vez mais comum a estimativa visual da altura em florestas nativas, com ou sem auxílio de uma régua encostada na árvore para fins de comparação (SILVA et al, 2012a). Estudos realizados em diferentes formações florestais demonstraram que o uso da estimativa visual para a medição da altura de árvores pode apresentar resultados confiáveis (GONÇALVES et al., 2009; 
SILVA et al., 2012a; CURTO et al., 2013; ANDRADE et al., 2016; SILVA et al., 2019).

A validação do uso da estimativa visual para a medição da altura de árvores em florestas nativas pode acarretar na redução dos custos de inventário, na medida em que o método se caracteriza como rápido e prático, podendo inclusive dispensar investimentos em instrumentos. Curto et al. (2013) observaram que as estimativas de altura de árvores pelo método de estimação visual, em geral, consumem um tempo muito baixo em relação aos métodos que utilizam algum instrumento de medição. Silva et al. (2019) perceberam que o tempo para a estimativa da altura pelo método visual é quase que instantâneo e consideraram que, em Floresta Ombrófila Aberta, o método de estimação visual com experiência resultou em menor custo para implementação e execução.

Busca-se, na execução de inventários florestais, métodos que apresentam menor erro e custo, os quais estão diretamente ligados ao tempo de medição de suas variáveis. Portanto, para resultados mais precisos e menos dispendiosos, devem ser adotados métodos adequados às características da população estudada (CESARO et al., 1994). Assim, este trabalho teve como objetivo comparar o método empírico da estimativa visual em relação ao hipsômetro digital Haglof para medição de altura de árvores em um fragmento de Floresta Estacional Decidual.

\section{MATERIAL E MÉTODOS}

\subsection{Caracterização da área}

O estudo foi realizado em um fragmento florestal denominado Mata do Olho d'Água, localizado no município de Macaíba, Mesorregião Leste do estado do Rio Grande do Norte, entre as coordenadas centrais $5^{\circ} 53^{\prime} \mathrm{S}$ e $35^{\circ} 23^{\prime} \mathrm{W}$, apresentando altitude média de $40 \mathrm{~m}$ e cerca de 270 ha. A área onde está localizado o fragmento compõe a Reserva Florestal da Escola Agrícola de Jundiaí, Unidade Acadêmica Especializada em Ciências Agrárias, da Universidade Federal do Rio Grande do Norte.

O clima local, de acordo com a classificação climática de Köppen, é do tipo As, ou seja, com estação chuvosa concentrada entre os meses de maio e julho, enquanto a estação seca ocorre entre os meses de setembro e dezembro. A precipitação média anual é de aproximadamente $1.280 \mathrm{~mm}$ e a temperatura média varia entre 24 e $28^{\circ} \mathrm{C}$ (ALVARES et al., 2013).

O fragmento é classificado como Floresta Estacional Decidual das Terras Baixas, o qual representa um ecótono entre a Floresta Estacional Semidecidual (Floresta Tropical Subcaducifólia) e a Savana-Estépica (Caatinga do Sertão), apresentando mais de $80 \%$ dos indivíduos desprovidos de suas folhas na época desfavorável do ano (CESTARO; SOARES, 2004; IBGE, 2012).

As espécies que ocorrem na área de estudo são comumente encontradas em formações florestais dos domínios Atlântico, Caatinga e Cerrado. Em função disso, observa-se no fragmento elevada diversidade de espécies, sendo encontrados indivíduos de grande e pequeno porte, com alguns apresentando muitos fustes (CESTARO; SOARES, 2004).

\subsection{Coleta de dados}

A coleta de dados foi realizada em parcelas permanentes instaladas entre novembro de 2017 e setembro de 2018, quando foram mensurados e plaqueteados todos os indivíduos vivos com circunferência a $1,30 \mathrm{~m}$ do solo (CAP) $\geq 10 \mathrm{~cm}$, utilizando fita métrica graduada em centímetros.

Dentre as parcelas permanentes instaladas no fragmento, foram selecionadas quatro parcelas, localizadas em área com relevo plano, nas quais foram amostrados 187 indivíduos, distribuídos em 23 espécies (Tabela 1).

Tabela 1. Listagem das espécies arbóreas amostradas no fragmento de Floresta Estacional Decidual localizado no município de Macaíba, Rio Grande do Norte.

Table 1. List of tree species sampled in the fragment of Deciduous Seasonal Forest located in the municipality of Macaíba, Rio Grande do Norte.

\begin{tabular}{lc}
\hline Espécies & $\mathrm{N}^{\circ}$ indivíduos \\
\hline Albizia polycephala (Benth.) Killip & 6 \\
Anadenanthera colubrina (Vell.) Brenan & 6 \\
Baubinia cheilantha (Bong.) Steud. & 4 \\
Cenostigma nordestinum E. Gagnon \& G.P. Lewis & 19 \\
Campomanesia dichotoma (O. Berg) Mattos & 1 \\
Casearia commersoniana Cambess. & 2 \\
Casearia decandra Jacq. & 20 \\
Casearia sylvestris Sw. & 9 \\
Ceiba glaziovii (Kuntze) K. Schum. & 1 \\
Croton blanchetianus Baill. & 2 \\
Cynophalla flexuosa (L.) J.Presl & 6 \\
Cynophalla hastata (Jacq.) J.Presl & 4 \\
Eugenia luschnatbiana (O.Berg) Klotzsch ex B.D.Jacks & 2 \\
Genipa americana L. & 1 \\
Guapira laxa (Netto) Furlan & 12 \\
Guettarda platypoda DC. & 1 \\
Handroanthus impetiginosus (Mart. ex DC.) Mattos & 21 \\
Libidibia ferrea (Mart. ex Tul.) L.P.Queiroz & 13 \\
Spondias mombin L. & 1 \\
Tabebuia roseoalba (Ridl.) Sandwith & 7 \\
Xylosma ciliatifolia (Clos) Eichler & 1 \\
Zanthoxylum syncarpum Tul. & 11 \\
Ziziphus joazeiro Mart. & 3 \\
NI & 34 \\
\hline Total & 187 \\
\hline NI = Não identificadas. &
\end{tabular}

\subsection{Métodos avaliados}

Os métodos avaliados para a estimação da altura das árvores foram os seguintes: 1) Estimativa visual, com balizamento de 3 metros; e 2) Estimativa com hipsômetro digital Haglof.

O método 1 compreendeu a estimação visual com auxílio de uma régua graduada de $3 \mathrm{~m}$ encostada às árvores para criar uma referência (parâmetro de comparação) ao mensurador, sem haver uma distância estabelecida entre o mensurador e as árvores observadas. O método 2 compreendeu o emprego do hipsômetro digital da marca Haglof, baseado no princípio trigonométrico para estimar a altura de árvores tomando como referência a distância entre o mensurador e o objeto (medida manualmente com trena) e dois ângulos, sendo o primeiro tomado mirando-se na base da árvore e o segundo mirando-se no ápice da árvore. $\mathrm{A}$ distância entre o mensurador e as árvores foi de 20 metros. O hipsômetro Haglof calcula a altura, que é mostrada no visor, não necessitando de cálculos. É importante ressaltar que cada método avaliado foi realizado por um mensurador, não havendo comunicação entre os dois para que não houvesse influência nos resultados. 


\subsection{Análise de dados}

A análise comparativa dos resultados foi realizada a partir da distribuição das alturas em classes. O número e o intervalo de classes foram definidos pelo método de Sturges. Assim, definiu-se as classes de altura em: 1) $<3,5 \mathrm{~m}, 2) 3,5<5 \mathrm{~m}, 3$ ) $5<6,5 \mathrm{~m}, 4) 6,5<8 \mathrm{~m}, 5) 8<9,5 \mathrm{~m}, 6) 9,5<11 \mathrm{~m}, 7) 11<$ $12,5 \mathrm{~m}, 8) 12,5<14 \mathrm{~m} \mathrm{e} 9)>14 \mathrm{~m}$. Também foram calculados os volumes cilíndricos individuais das árvores $(\mathrm{V}=$ $\left.\left(\pi * \mathrm{DAP}^{2} / 4\right) * \mathrm{~h}\right)$, que foram distribuídos em classes diamétricas, para fins de análise comparativa.

Os dados foram tabulados no software Microsoft Excel e analisados no software BioEstat ${ }^{\circledR}$ versão 5.3. Primeiramente, realizou o teste de normalidade de Lilliefors. Como os dados apresentaram distribuição não paramétrica, foram submetidos a análise de variância de Kruskal-Wallis em nível de $5 \%$ de significância e, quando apresentaram diferença estatística, as médias foram comparadas pelo teste de Duun também ao nível de 5\% de probabilidade.

\section{RESULTADOS}

A Figura 1 apresenta a relação entre os diâmetros tomados à $1,30 \mathrm{~m}$ de altura (DAP) e as alturas das árvores, estimadas pelos dois métodos. Com base nas linhas de tendência, observa-se que as alturas estimadas pelos dois métodos foram bastante semelhantes para as árvores com $\mathrm{DAP} \leq 15 \mathrm{~cm}$. No entanto, ao observar as árvores com mais de $15 \mathrm{~cm}$ de DAP, nota-se que houve aumento da diferença entre os métodos, havendo uma subestimação da altura pelo método visual com balizamento em relação ao hipsômetro digital.

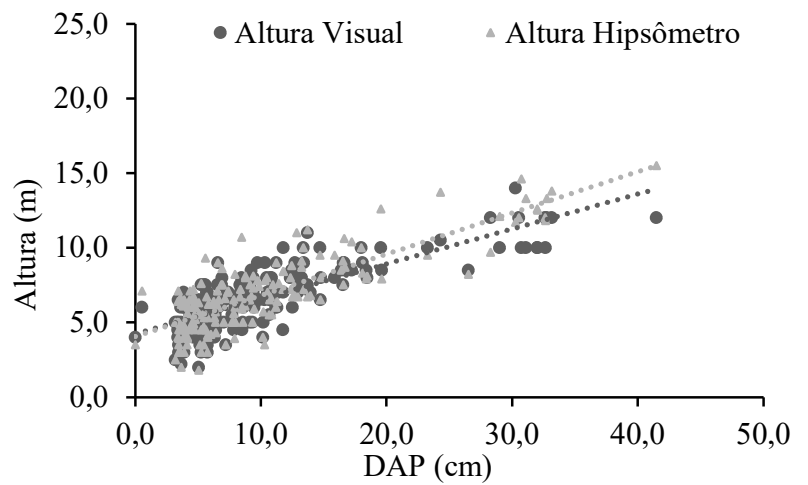

Figura 1. Relação entre o DAP e altura estimada das árvores pela estimativa visual com balizamento e hipsômetro digital em um fragmento de Floresta Estacional Decidual.

Figure 1. Relationship between DAP and estimated height of trees by visual estimation with beacon and digital hypsometer in a fragment of Seasonal Deciduous Forest.

As alturas mínimas encontradas por meio da estimativa visual com balizamento e do hipsômetro digital foram, respectivamente, 2,00 e $1,78 \mathrm{~m}$, enquanto que as máximas foram, respectivamente, 14,00 e $15,50 \mathrm{~m}$. A altura média das árvores com o método de estimativa visual com balizamento foi inferior $(6,57 \mathrm{~m})$ à do hipsômetro digital $(6,80 \mathrm{~m})$.

$O$ teste de médias confirmou que houve diferença estatística nas classes 4) $6,5<8 \mathrm{~m} \mathrm{e} \mathrm{8)} 12,5<14 \mathrm{~m}$. Os dois métodos se mostraram estatisticamente semelhantes para as demais classes de altura (Tabela 1). Ao comparar os volumes cilíndricos individuais estimados em classes diamétricas, houve diferença estatística apenas na classe 28,98<33,28 cm.
Nessa classe de DAP, todas as árvores pertenciam às classes de altura 7) $11<12,5 \mathrm{~m}$ e 8) $12,5<14 \mathrm{~m}$. Portanto, fica evidente que a subestimação das alturas pela estimativa visual com balizamento influenciou diretamente na estimativa de volume de árvores maiores (Figura 2).

Tabela 1. Altura média estimada das árvores pela estimativa visual com balizamento e hipsômetro digital por classes de altura em um fragmento de Floresta Estacional Decidual.

Table 1. Estimated average height of trees by visual estimation with beacon and digital hypsometer by height classes in a fragment of Seasonal Deciduous Forest.

\begin{tabular}{ccc}
\hline Classes & Estimativa visual & Hipsômetro digital \\
\hline 1 & $2,98 \mathrm{a}$ & $3,05 \mathrm{a}$ \\
2 & $4,56 \mathrm{a}$ & $4,53 \mathrm{a}$ \\
3 & $6,14 \mathrm{a}$ & $6,05 \mathrm{a}$ \\
4 & $7,46 \mathrm{a}$ & $7,22 \mathrm{~b}$ \\
5 & $8,76 \mathrm{a}$ & $8,72 \mathrm{a}$ \\
6 & $10,11 \mathrm{a}$ & $10,34 \mathrm{a}$ \\
7 & $12,00 \mathrm{a}$ & $11,90 \mathrm{a}$ \\
8 & $14,00 \mathrm{a}$ & $13,22 \mathrm{~b}$ \\
9 & - & 15,05 \\
\hline
\end{tabular}

Médias seguidas por letras iguais, na linha, não diferem estatisticamente entre si pelo teste de Dunn ao nível de 5\% de probabilidade.

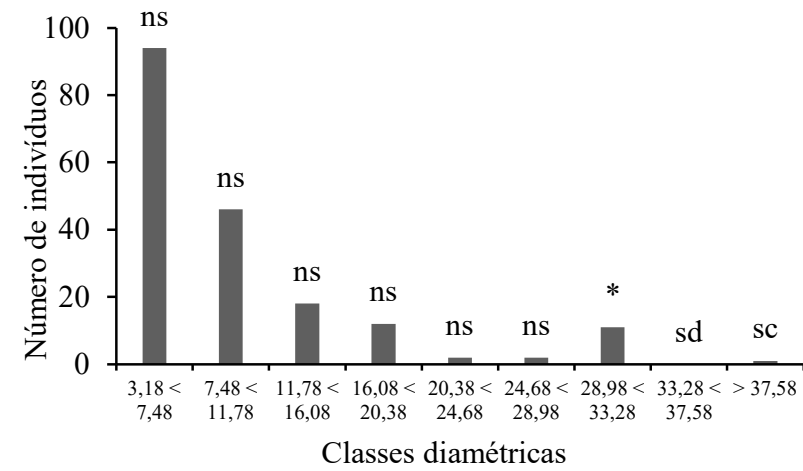

Figura 2. Distribuição da frequência total das árvores em classes diamétricas. Legenda: ns - não significativo, ${ }^{*}$ - apresenta diferença estatística, sd - sem dados para comparação, sc - sem comparação por haver apenas um indivíduo.

Figure 2. Distribution of the total frequency of the trees in diametric classes. Legend: ns - not significant, ${ }^{*}$ - shows statistical difference, sd - without data for comparison, sc - without comparison because there is only one individual.

\section{DISCUSSÃO}

A diferença observada entre os métodos utilizados para a mensuração da altura demonstrou que conforme aumentou o DAP, as árvores tenderam a apresentar maiores alturas, dificultando a estimativa visual, devido ao aumento da distância entre a régua utilizada para comparação e o topo da árvore. Em função disso, observou-se uma subestimação mais acentuada da estimativa visual em árvores com as maiores alturas.

Curto et al. (2013) avaliando a precisão e o desempenho do hipsômetro Vertex, do clinômetro digital, da estimativa visual com auxílio de régua e da estimativa visual com e sem treinamento para a mensuração da altura em um fragmento de Floresta Estacional Semidecidual, observaram que para as árvores de pequeno porte os métodos avaliados apresentaram estimativas mais confiáveis, enquanto os valores obtidos para as árvores de maior porte foram menos confiáveis. Vale salientar, que ambos os métodos de 
estimação visual diferiram da altura real observada para as árvores de maior porte, ou seja, $>21 \mathrm{~m}$, o que confirma a dificuldade de se estimar visualmente a altura das árvores mais altas, mesmo com treinamento ou tendo uma referência como auxílio, corroborando com os resultados encontrados neste trabalho.

Ao avaliarem métodos de mensuração de altura em florestas naturais, os quais incluíam a estimativa visual com e sem treinamento e a estimação com hipsômetro Vertex, Silva et al. (2012a) observaram que o aumento da altura da árvore comprometeu a precisão da estimação em todos os métodos avaliados, sendo mais evidente na estimação visual sem treinamento. Os autores destacaram os métodos de estimação de altura avaliados por eles apresentaram estimativas mais confiáveis para a menor classe de altura (15$25 \mathrm{~m})$ e menos confiáveis para a maior classe de altura $(>30$ $\mathrm{m})$.

Resultados semelhantes foram encontrados por Silva et al. (2019) ao analisarem diferentes métodos indiretos de medição da altura comercial de árvores na região Amazônica, os quais observaram uma tendência da estimação visual em subestimar os valores da altura comercial das árvores. Os autores indicaram a utilização do clinômetro Suunto, devido a exatidão das medidas de altura obtidas, em conjunto com a estimativa visual realizada por uma pessoa experiente, por apresentar menor tempo de execução e baixo custo de implementação.

Curto et al. (2013), discutiram que a tendência do método de estimativa visual em subestimar a altura de árvores com maior porte, em princípio, é algo difícil de explicar, mas pode estar associada ao receio do mensurador de exagerar na medição da altura. No entanto, os autores ressaltaram que é algo muito subjetivo, podendo variar de pessoa para pessoa, sendo um dos grandes inconvenientes da utilização desse método.

Em trabalho realizado na Amazônia, Gonçalves et al. (2009) não encontraram diferença estatística na altura média de árvores estimadas visualmente com mensurador experiente e com instrumento a laser. Apesar disso, os autores observaram limitações quanto ao uso do aparelho em floresta tropical, principalmente, devido a restrições de visibilidade, tendo em vista que não foi possível realizar as medições de $51,2 \%$ do total de árvores amostradas com o aparelho, enquanto a estimativa visual da altura foi realizada em mais de $99 \%$ do total de árvores amostradas.

David (2011), ao comparar o uso do Haglof e régua telescópica observou que os valores obtidos eram estatisticamente iguais, assim como parte dos resultados deste trabalho, tendo em vista que não foi observada diferença estatística entre os métodos utilizados para árvores abaixo de $11 \mathrm{~m}$ de altura.

Enquanto Andrade et al. (2016), avaliando dados coletados em área de Cerrado sensu stricto, encontraram diferença estatística entre os métodos utilizados para a medição da altura de árvores dentro de todas as classes de altura $(\mathrm{h}<7 \mathrm{~m} ; 7<\mathrm{h}<9 \mathrm{~m}$; e $\mathrm{h}>9 \mathrm{~m})$. Para os autores, a estimativa visual mostrou-se adequada somente para medir a altura total de árvores maiores que $7 \mathrm{~m}$, diferindo de outros trabalhos e dos resultados obtidos neste trabalho.

A partir da análise dos resultados deste trabalho, constatou-se que a utilização do hipsômetro digital para a mensuração da altura de árvores de maior porte proporcionou resultados mais confiáveis para a estimativa de volume. Pois, conforme Jesus et al. (2012), Silva et al. (2012b) e Feliciano et al. (2016), os hipsômetros disponíveis no mercado apresentam excelente precisão e pouco diferem entre si.

Apesar disso, deve-se considerar que a estimativa visual com experiência está entre os métodos com menor custo de implementação e tempo de execução (SILVA et al., 2019). Feliciano et al. (2016) acrescentaram que, para a obtenção de excelente precisão ao utilizar hipsômetros, os operadores precisam de um bom treinamento, visando o manuseio adequado dos aparelhos, visto que quanto maior a altura da árvore, menor se torna a chance de visualização do seu topo, aumentando a probabilidade de subestimação ou superestimação.

Nesse sentido, é importante destacar no uso de hipsômetros que a distância previamente fixada entre o operador e a árvore, geralmente entre 15 e $20 \mathrm{~m}$, muitas vezes pode não ser adequada para a melhor visualização da copa. Para estimativas visuais, não há necessidade de cumprimento de distâncias determinadas e o mensurador tem a liberdade de escolher aquela que garante a melhor visualização. Isso acelera o processo de mensuração e, consequentemente, acarreta na redução dos custos do inventário. Porém, independentemente do método, o treinamento é indispensável para garantir confiabilidade.

\section{CONCLUSÃO}

A estimativa visual com balizamento pode ser utilizada com confiabilidade para a medição da altura de árvores de menor porte em Floresta Estacional Decidual, pois há uma tendência de subestimação da altura de árvores maiores. Para árvores de maior porte, com altura acima de $11 \mathrm{~m}$, o hipsômetro digital mostrou-se mais confiável.

\section{REFERENCES}

ALVARES, C. A; STAPE, J. L; SENTELHAS, P. C; GONÇALVES, J. L de M; SPAROVEK, G. Köppen's climate classification map for Brazil. Meteorologische Zeitschrift, v. 22, n. 6, p. 711-728, 2013. DOI: https://doi.org/10.1127/0941-2948/2013/0507

ANDRADE, V. C. L.; RIBEIRO, J. R.; PINTO, I. O.; SANTOS, M. J. F.; TELES, L. B.; TERRA, D. L. C. V. Hipsômetros baseados no princípio geométrico avaliados em área de Cerrado sensu stricto. Nativa, Sinop, v. 4, n. 5, p. 333-336, 2016. DOI: http://dx.doi.org/10.14583/2318-7670.v04n05a10

CESARO, A.; ENGEL, O. A.; FINGER, C. A. G.; SCHNEIDER, P. R. Comparação dos métodos de amostragem de área fixa, relascopia, e de seis árvores, quanto a eficiência, no inventário florestal de um povoamento de Pinus sp. Ciência Florestal, Santa Maria, v. 4, n. 1, p. 98-108, 1994.

CESTARO, L. A; SOARES, J. J. Variações florística e estrutural e relações fitogeográficas de um fragmento de floresta decídua no Rio Grande do Norte, Brasil. Acta Botanica Brasilica, Brasília, v. 18, n. 2, p. 203-218, 2004. https://doi.org/10.1590/S0102-33062004000200001

CURTO, R. A; SILVA, G. F.; SOARES, C. P. B; MARTINS, L. T; DAVID, H. C. Métodos de estimação de altura de árvores em floresta estacional semidecidual. Floresta, Curitiba, v. 43, n. 1, p. 105-116, 2013. DOI: http://dx.doi.org/10.5380/rf.v43i1.26791 
DAVID, H. C. Métodos de medição de altura de árvores em uma floresta nativa do sul do Espírito Santo. 2011. 24 f. TCC (Graduação) - Curso de Engenharia Florestal, Universidade Federal do Espírito Santo, Jerônimo Monteiro, 2011.

FELICIANO, M. E.; RIBEIRO, A; FERRAZ FILHO, A. C; VITOR, P. C. G. Avaliação de diferentes hipsômetros na estimativa da altura total. Revista Verde de Agroecologia e Desenvolvimento Sustentável, Pombal, v. 11, n. 2, p. 1-5, 2016. DOI: https://doi.org/10.18378/rvads.v11i2.3741

GONÇALVES, D. A.; VAN ELDIK, T.; POKORNY, B. O uso de dendrômetro a laser em Florestas Tropicais: Aplicações para o manejo florestal na Amazônia. Floresta, Curitiba, v. 39, n. 1, p. 175-187, 2009. DOI: http://dx.doi.org/10.5380/rf.v39i1.13737

INSTITUTO BRASILEIRO DE GEOGRAFIA E ESTATÍSTICA - IBGE - Manual Técnico da Vegetação Brasileira. 2. ed. Rio de Janeiro: IBGE, 2012.

JESUS, C. M. D.; MIGUEL, E. P.; LEAL, F. A.; ENCINAS, J. I. Avaliação de diferentes hipsômetros para medição da altura total em um povoamento clonal de Eucalyptus urophylla x Eucalyptus grandis. Enciclopédia Biosfera, Goiânia, v. 8, n. 15, p. 291-299, 2012.

SILVA, J. C.; MENDONCA, A. R.; SILVA, G. F.; CURTO, R. A.; FIGUEIREDO, L. T. M.; SILVA, M. L. M. Métodos de medição da altura comercial de árvores na região Amazônica. Scientia Forestalis, Piracicaba, v. 47, n. 123 , p. 588-598, 2019. DOI: http://dx.doi.org/10.18671/scifor.v47n123.20

SILVA, G. F.; MÔRA, R.; CURTO, R. A. Simulação de erros na medição de altura de árvores inclinadas com aparelhos baseados em princípios trigonométricos. Nativa, Sinop, v. 5, n. 5, p. 372-379, 2017. DOI: http://dx.doi.org/10.5935/2318-7670.v05n05a12

SILVA, G. F.; CURTO, R. A; SOARES, C. P. B; PIASSI, L. C. Avaliação de métodos de medição de altura em florestas naturais. Revista Árvore, Viçosa, v. 36, n. 2, p. 341-348, 2012a. DOI: http://doi.org./10.1590/S010067622012000200015

SILVA, G. F.; OLIVEIRA, O. M.; SOUZA, C. A .M; SOARES, C. P. B; LEMOS, R. Influência de diferentes fontes de erro sobre as medições de alturas de árvores. Cerne, Lavras, v. 18, n. 3, p. 397-405, 2012 b. https://doi.org/10.1590/S0104-77602012000300006 